\title{
Simulations of charged droplet collisions in shear flow
}

\author{
Orest Shardt $^{\mathrm{a}, *}$, Sushanta K. Mitra ${ }^{\mathrm{b}}$, J. J. Derksen ${ }^{\mathrm{a}}$ \\ ${ }^{a}$ Department of Chemical and Materials Engineering, University of Alberta, Edmonton, Alberta, Canada \\ ${ }^{b}$ Department of Mechanical Engineering, Lassonde School of Engineering, York University, Toronto, Canada
}

\begin{abstract}
Collisions of charged droplets in the shear flow of an electrolyte were simulated to investigate the effects of surface charges and double layers on the critical conditions for coalescence. A lattice Boltzmann method phase field flow solver was coupled with an iterative finite-difference solver for the linearized Poisson-Boltzmann equation describing electric double layers. The simulations resolve both the phase field diffuse interface and electric double layer. The critical capillary numbers for coalescence were determined under varying strengths of the electric interactions. Critical capillary numbers between 0.03 and 0.2 were found for droplet radii spanning 25 to 50 lattice nodes (12.5 to 25 times the characteristic interface thickness), with lower critical values for larger droplets. The droplet interfaces had a constant potential. Once electric repulsion becomes comparable to the viscous shear force on the drops, the critical capillary numbers decrease, and the decrease is smaller for longer Debye lengths. Though the ratio of droplet size and Debye length that can be achieved in the simulations is constrained by high computational demands, the simulations provide insight into the effects of surface charge on the interactions between interfaces in multiphase flows.
\end{abstract}

Keywords: surface charge, droplet, coalescence, Poisson-Boltzmann equation, shear flow, simulation, lattice Boltzmann method

\footnotetext{
*Corresponding author

Email addresses: shardt@ualberta.ca (Orest Shardt), mitras@yorku . ca (Sushanta K. Mitra), jos@ualberta.ca (J. J. Derksen)
} 


\section{Introduction}

Interfaces between fluids are often charged for a variety of reasons[1], such as adsorption of ions and dissociation of acidic groups, and these charges give rise to a wide range of phenomena. For example, air bubbles[2, 3] and oil drops in water[4-6] have a negative charge that depends on the $\mathrm{pH}$ of the electrolyte solution they are in. Because of the charge on their surface and the diffuse charge in the electrolyte around them, drops move when external fields are applied[7-11]. Attractive and repulsive forces due to electric interactions between charges on interfaces and ions in the fluid also play an important role in the stability of emulsions against coalescence[1]. In the classic DLVO (Derjaguin and Landau [12] and Verwey and Overbeek [13]) theory, the balance between van der Waals attraction and electric attraction/repulsion on droplets and particles is used to assess the stability of emulsions and particle suspensions. Interactions between interfaces are not limited to electric and van der Waals forces[1]; for example many types of surfactants are used to control interfacial interactions, and they may have molecular structures, such as long hydrocarbon chains, that allow them to interact over longer distances. As a further complication, the distribution of surfactants and charges on an interface may not be uniform[14, 15]. Furthermore, variations in surface tension on interfaces give rise to Marangoni stresses and flows that alter the motion of droplets[16] and the flow between them during collisions[17].

Despite the important role of charge in the interactions between interfaces, simulations of multiphase flow rarely include electric phenomena. In fact, the details of interfacial interactions are usually neglected due to the already considerable computational expense of simulating the flow. In typical simulations, interfaces merge and threads break when their size falls below the resolution of the simulation or a specified critical size[18-20]. Since small changes in composition, such as addition of a surfactant or a change in $\mathrm{pH}$, can have a profound effect on macroscopic characteristics of multiphase flows, there is a need to account for the different interactions between interfaces that determine the conditions for the coalescence and breakup of droplets. The ability to perform such detailed simulations of multiphase flows is relevant to studies of droplet size distributions in turbulent mixing[21, 22] and the behaviour of droplets in micro-scale devices[23]. Working towards this goal, we have coupled a multiphase flow solver with a solver for electrostatic potential 
to simulate collisions of deforming charged droplets. To our knowledge, simulations of moving charged interfaces and collisions of charged droplets have not been previously reported in the open literature.

In general, colliding pairs of droplets coalesce unless their capillary number (ratio of viscous and interfacial forces) exceeds a critical value. The critical conditions for coalescence of uncharged droplets in shear flow have been previously studied in experiments with polymers[24-29] and simulations[30, 31] with the free-energy binary-liquid lattice Boltzmann method (LBM)[32]. This phase field type method is straightforward to implement on parallel computing architectures, in particular graphics processing units (GPUs) whose improved performance relative to conventional processors allowed highly-resolved simulations with droplet radii up to 200 lattice nodes[31]. The droplets' diffuse interfaces were sufficiently resolved that the merging of adjacent interfaces was determined by the dynamics of the phase field rather than a resolution limit. In the phase field method used in these previous simulations, nearby interfaces experience attraction due to a negative disjoining pressure whose dependence on the distance between the interfaces is similar to that for van der Waals interactions[33]. As in physical systems, a positive disjoining pressure is required in simulations to stabilize films against coalescence. Recently, Dollet et al. [34] used a lattice Boltzmann method that combines short-range attraction and longer-range repulsion to simulate a stable foam. We now add electrostatic forces and charged interfaces to the phase field model used in previous simulations of droplets with clean interfaces. The resulting coupled phase field and electrostatics model we present is analogous to DLVO theory with attractive van der Waals interactions and repulsive electrostatic interactions.

While Matsuyama et al. [35] studied the Rayleigh instability[36] of a charged drop, most previous simulations of coupled flow and electric phenomena with LBM have considered the effects of external fields on diffuse charges and interfaces between dielectric media (droplets and their surrounding fluid). For example, Wang et al. [37], Wang and Kang [38], and Lin and Chen [39] used coupled lattice Boltzmann methods to study electroosmotic flow, while Hlushkou et al. [40] coupled a lattice Boltzmann flow solver with a finite difference solver for the electric potential. Wang et al. [41] studied electroosmotic flow in porous media. The effects of external fields on multiphase 
flows with interfaces between (leaky) dielectrics have been simulated with the volume-of-fluid[42], level set[43], and phase field[44] methods. Various authors have examined the modelling of ion free energies in solution[45], and the inclusion of ion free energies into phase field models of liquid mixtures[46, 47], including lattice Boltzmann methods[48], but dynamic simulations of droplet motion in electrolytes with such models have not been reported.

In the sections that follow we describe the relevant theory of electrokinetic phenomena and the numerical methods that have been combined to form the coupled multiphase flow and electrostatic potential solver. We then assess the accuracy of the solver through several benchmarks. Finally, we present and interpret the effects of surface charge on the critical conditions for coalescence of a droplet pair in simple shear flow.

\section{Theory and Numerical Models}

The simulation method consists of two parts: a multiphase flow solver and a potential solver. Both solvers have been implemented on GPUs. In this work, we use only one GPU, though the methods may also be implemented on clusters of GPUs as has been done for the flow solver[30, 31 .

\subsection{Multiphase flow}

The multiphase flow solver provides the solution to the incompressible Navier-Stokes equations, consisting of a mass balance and the momentum balance

$$
\frac{\partial \vec{u}}{\partial t}+(\vec{u} \cdot \nabla) \vec{u}=-\frac{1}{\rho} \nabla P+v \nabla^{2} \vec{u}+\frac{1}{\rho} \vec{b}
$$

where $\vec{u}$ is the flow velocity, $\rho$ is the fluid density, $P$ is the pressure, $v$ is the fluid kinematic viscosity, and $\vec{b}$ is the sum of any external forces (per unit volume) acting on the fluid. For initial simplicity, we consider a pair of fluids with equal densities and viscosities. The composition of the fluid mixture is tracked by the order parameter $\phi$, which varies between -1 and +1 , and evolves according to the advection-diffusion equation

$$
\frac{\partial \phi}{\partial t}+\nabla \cdot(\phi \vec{u})=M \nabla^{2} \mu_{\phi}
$$

In Eq. 2, $\mu_{\phi}$ is the local chemical potential of the fluid that depends on the composition $\phi$ and its gradient $\nabla \phi$. The mobility of the phase field is $M$. We solve this system of equations with the 
binary-liquid free-energy lattice Boltzmann method[32]. Both the flow and phase field evolution are solved in three dimensions with LBM density distributions that have 19 discrete directions. The phase field is coupled bidirectionally with the flow: the velocity in Eq. 2 is obtained from the flow field and the pressure tensor in the momentum balance (Eq. 1) is modified to include a thermodynamic component. The resulting pressure is

$$
P=\frac{1}{3} \rho-\frac{1}{2} A \phi^{2}+\frac{3}{4} A \phi^{4}-\kappa_{\phi} \phi \nabla^{2} \phi-\frac{1}{2} \kappa_{\phi} \nabla \phi \cdot \nabla \phi
$$

where $A$ and $\kappa_{\phi}$ are parameters of the free-energy model. Due to the use of a free energy with two minima, specifically the free energy functional $F[\phi(\vec{x})]$

$$
F=\int_{V}\left[\frac{1}{3} \rho \ln \rho+\frac{A}{2} \phi^{2}\left(\frac{1}{2} \phi^{2}-1\right)+\frac{\kappa_{\phi}}{2}(\nabla \phi \cdot \nabla \phi)\right] \mathrm{d} V,
$$

the fluid mixture separates into regions with $\phi= \pm 1$ and interfaces between regions have an excess energy (interfacial tension)[32] $\sigma=\frac{2 \sqrt{2}}{3} \sqrt{\kappa_{\phi} A}$. The transition in composition across interfaces is smooth, has a characteristic length[32] $\ell_{\phi}=\sqrt{2 \kappa_{\phi} / A}$, and 99\% of the change occurs over the distance $5.3 \ell_{\phi}$ that may be considered the thickness of the interface. The body force $\vec{b}$ is included in the collision operation of the LBM using the method of He et al. [49]. We note that including the term $\vec{b} \Delta t /(2 \rho)$ in the calculation of the physical flow velocity (see e.g. Pooley and Furtado [50]) is essential for the simulations of electroosmotic flow in which the force is highest where the flow speed is slowest.

In this work, we use the same code to examine both single phase benchmark problems and two phase simulations with suspended droplets. For the single phase flow simulations, we initialize $\phi$ to -1 throughout the domain. In the simulations of sheared droplet pairs, $\phi$ is initialized to 1 inside the drops and -1 outside. We allow the interface to smooth out for 100 time steps before starting the shear flow and applying the electrostatic body force.

For the purposes of this paper, we consider the LB method to provide the velocity, pressure, and phase fields that describe the evolution of the system. The details of the specific method used, and in general the use of lattice Boltzmann methods for computing flows, are available in a wide variety of sources[32, 50-52]. The free-energy LBM we employ has been used to study, for example, droplet formation in microchannels[53] and droplet deformation and breakup in shear[54]. We do 
not describe the free-energy LBM in further detail, and instead turn our attention to the modelling of electrokinetic phenomena. These effects are coupled with the multiphase flow solver through the body force $\vec{b}$. The nature of the body force, which depends on the electrokinetic phenomena being considered, is described in the next section.

\subsection{Electrokinetics}

The literature on electrokinetic phenomena, which are flow phenomena that occur due to charge, is extensive and has a long history[55]. Near charged surfaces or interfaces, the distribution of ions is determined by the balance between the electrostatic forces on them and their thermal diffusion. The result is an electric double layer (EDL) in which ions with the same charge as the interface are repelled from it, and ions with the opposite charge are attracted. The net charge in the EDL is opposite to the charge of the interface, and it decays with increasing distance from the interface. In electro-neutral systems, the total charges on the interface and in the electrolyte balance. The Poisson equation

$$
\varepsilon \nabla^{2} \psi=-\rho_{e}
$$

relates the electrostatic potential $\psi$ to the free charge density $\rho_{e}$ in a linear dielectric material with permittivity $\varepsilon[1]$. At equilibrium, the concentration of ions is related to the electrostatic potential through the Boltzmann distribution

$$
\eta_{i}=\eta_{i \infty} \exp \left(-\frac{z_{i} e \psi}{k_{\mathrm{B}} T}\right)
$$

where $\eta_{i}$ is the number density (number per unit volume) of the $i^{\text {th }}$ charged component, $\eta_{i \infty}$ is the density in the bulk (far from the interface), $z_{i}$ is the valence, $e$ is the elementary charge, $k_{\mathrm{B}}$ is the Boltzmann constant, and $T$ is the absolute temperature[1]. We restrict our attention to symmetric $z: z$ electrolytes, with components denoted by $i=+,-$, for which $\eta_{+\infty}=\eta_{-\infty} \equiv \eta_{\infty}$. It follows that the net free charge density is

$$
\rho_{e}=e z\left(\eta_{+}-\eta_{-}\right)=-2 e z \eta_{\infty} \sinh \frac{z e \psi}{k_{\mathrm{B}} T}
$$

For $\frac{z e \psi}{k_{\mathrm{B}} T}$ sufficiently small, the hyperbolic sine may be linearized as $\sinh \frac{z e \psi}{k_{\mathrm{B}} T} \approx \frac{z e \psi}{k_{\mathrm{B}} T}$. Using this simplification, which is called the Debye-Hückel approximation, and combining Eqs. 5 and 7 
yields the linearized Poisson-Boltzmann equation:

$$
\nabla^{2} \psi=\kappa^{2} \psi
$$

where

$$
\kappa^{2}=\frac{2 e^{2} z^{2} \eta_{\infty}}{\varepsilon k_{\mathrm{B}} T}
$$

The Debye length $\kappa^{-1}$ characterizes the thickness of the EDL. We note that under the DebyeHückel approximation, $\rho_{e}=-\varepsilon \kappa^{2} \psi$, a form that simplifies computation of the free charge density by avoiding the evaluation of the Laplacian of the potential (Eq. 5).

Boundary conditions for the electrostatic potential are in general complex due to the adsorption and reaction phenomena that are responsible for surface charges[1]. Two limiting cases are commonly considered[56-58]: a prescribed surface charge or surface potential. We limit our scope to the latter case and consider only the zeta potential $\zeta$, which is the potential at the slip plane that separates the fluid from molecules that stay attached to the surface. Since we study systems that are characterized by only one zeta potential, we normalize the potential by $\zeta$ in the numerical solver. In simulations of electroosmotic flow, the zeta potential is imposed on the walls of the channel; in the droplet collision simulations, it is imposed on the liquid-liquid interface.

The electrostatics and flow solvers are coupled through the body force term in Eq. 1. The general form for the body force is

$$
\vec{b}=\rho_{e} \vec{E}
$$

where terms due to non-uniform dielectric permittivity and electrostriction have been omitted from the Korteweg-Helmholtz force density[1]. The electric field $\vec{E}$ depends on the problem being considered. For the electroosmotic flow benchmark it is the applied external field and the charge density is determined from the potential $\psi$. In the simulations of charged droplets, it is the electric field in the EDL, $-\nabla \psi$. For these simulations with only internal fields, we include the osmotic pressure of the ions in $P$ (Eq. 1). One may alternatively add an osmotic body force, the difference between the two methods being an irrotational body force that can be absorbed into a modified $P[59]$. For the droplets with constant surface potential that we simulate, the osmotic pressure at their surface is uniform and constant and does not contribute a net force on them[58]. 
We solve Eq. 8 on a uniform grid whose points are aligned with the nodes of the LBM flow solver. We discretize the Laplace operator in Eq. 8 with a 19 point stencil[50, 60]. This matches the stencil used to compute the Laplacian of the phase field in the free-energy LBM[50]. Discretization provides a system of linear equations, one per grid point, which are solved with the Jacobi iteration method implemented on a GPU. Though other methods, such as successive over-relaxation[61], converge in fewer iterations, the Jacobi method is straightforward to parallelize. At this time, we consider only the Dirichlet type boundary condition $\psi=\zeta$ at various locations in the domain, depending on the system being simulated. When required for the body force term, the gradient of $\psi$ is computed with a 10 point stencil[50].

In the simulations with moving droplet interfaces, we adopt several simplifying assumptions. We assume that the two fluids are perfect dielectrics with equal permittivity (leaky dielectrics have been previously coupled with phase field simulations[44]). No ions are present in the droplets, and we assume that the potential in the external electrolyte evolves quasi-statically. In other words, we assume that the charge distribution relaxes quickly to its equilibrium compared to the time scale of the flow. Without this assumption, a Nernst-Planck advection-diffusion equation would need to be solved for each ion. While this is a reasonable direction for future work, it is not pursued in this paper. At every timestep of the flow solver, we update the location of the interface. This is performed by setting $\psi / \zeta=1$ in all nodes with $\phi>0$ and $\psi / \zeta<1$ (arbitrarily chosen to be $\psi / \zeta=0.99$ ) in any nodes where $\phi \leq 0$ having previously been $>0$. We then iterate the finitedifference solver at all nodes with $\psi / \zeta<1$ until convergence. Due to the absence of free charge inside the drops and a fixed potential on their surface, the potential inside the drops is $\psi=\zeta$.

The body force in the droplet simulations is computed at each timestep from the updated potential field. The potential solver provides a continuous electrostatic potential field from which charge densities and electric fields can be computed. Nodes are classified into three groups that have different body forces. For bulk nodes (nodes with $\psi / \zeta<1$ ), the body force is $\vec{b}=\varepsilon \kappa^{2} \psi \nabla \psi$ (Eq. 10 with $\vec{E}=-\nabla \psi$ and $\rho_{e}$ from Eq. 5 with Eq. 8 used to eliminate $\nabla^{2} \psi$ ). Droplet nodes, which are nodes with $\psi / \zeta=1$ that are not adjacent to any nodes with $\psi / \zeta<1$, have $\vec{b}=0$. Nodes with $\psi / \zeta=1$ that are adjacent to at least one node with $\psi / \zeta<1$ are considered interface nodes. The 
force on a charged interface is the product of the charge density (per unit area) and an effective electric field that is the average of the internal and external electric fields[62], which may also be derived from the Maxwell stress[1] on a constant-potential surface. We spread the interfacial force to the volume occupied by each interfacial node by using the (volume) charge density computed with Eq. 5. The electric field is computed as $-\nabla \psi$ with a 10 point stencil that includes points inside (with $\psi / \zeta=1$ ) and outside the droplet. This gradient of the potential can be decomposed into components parallel and normal to the interface. Since $\psi / \zeta=1$ along the interface, the parallel components are zero, and the normal component is the net change across the interface, which is the desired average of the internal (zero) and external gradients.

As an alternative to coupling electrostatics with multiphase flow through a body force, the electrostatic free energy could be added to the free energy of the system[47,63] and coupled through a modified pressure tensor, like the coupling with the phase field. We expect that this alternative method would increase spurious currents[50], and we therefore use a body force.

\section{Results and Discussion}

\subsection{Benchmarks}

Before studying collisions of charged droplets in shear, we evaluated the accuracy of the electrostatic potential solver, the convergence of the coupled solver applied to transient single-phase electroosmotic flow, and the potential and hydrostatic pressure in and around a stationary drop. The accuracy and convergence benchmarks are described in the Supplementary Information. The accuracy of the potential solution was assessed for a square channel. Since Debye lengths larger than the interface thickness and smaller than the droplet radii are needed for the simulations of droplet collisions, two resolutions of the EDL (ratios of the Debye length and computational grid spacing) were considered: 15 and 25. The convergence criterion for the iterative linear solver was chosen to be a maximum change of $10^{-6}$ in the normalized potential. With the chosen resolutions of the EDL and termination criterion, the solution times were satisfactory and the maximum absolute errors (normalized by surface potential) between the numerical and exact solutions were low: $4 \times 10^{-4}$ to $2 \times 10^{-3}$. The transient electroosmotic flow benchmark showed second order convergence of the L2 error in the flow field at early and late times. With the two chosen Debye lengths 
(15 and 25), the relative error in the numerical solution for the maximum steady flow speed was at most $1 \%$. Considering the good accuracy of the potential and flow solvers for single-phase flow in a square channel, we now use the chosen parameters for the solvers in benchmarks with droplets.

\subsubsection{Stationary droplets}

In this section we evaluate the accuracy of the coupled solvers for stationary droplets. We assess the accuracy in three ways: the potential solution, the pressure field around a solid charged sphere, and the difference in pressure between the inside and outside of a droplet. We consider the two Debye lengths $\kappa^{-1}=15$ and 25. Droplets are implemented with a stair-stepped interface. The domains are cubic and periodic.

Figure 1 shows the potentials along two lines in cross-sections through a $128 \times 128 \times 128$ 1.u. domain with a 25 l.u. radius sphere. Solid lines indicate the exact potential outside a sphere of radius $R$ in an infinite domain:

$$
\psi(\vec{x})=\zeta \frac{R}{r} \exp (\kappa(R-r))
$$

where $r=\|\vec{x}\|$ is the distance of the point $\vec{x}=(x, y, z)$ from the centre of the sphere $(\vec{x}=\overrightarrow{0})$. The numerical (periodic domain) and exact (infinite domain) solutions agree well until a distance of one radius from the surface ( $2 R$ from the centre). The computed potentials along the two lines then deviate from the exact solution, with greater deviation along the line to the closest boundary $(y=0)$. The difference between the numerical and exact potentials is larger, as expected, for the longer Debye length.

From the Navier-Stokes momentum balance (Eq. 1), the static pressure with the body force $\vec{b}=\varepsilon \kappa^{2} \psi \nabla \psi$ satisfies

$$
\nabla P=\varepsilon \kappa^{2} \psi \nabla \psi
$$

which may be rewritten as

$$
\nabla P=\frac{1}{2} \varepsilon \kappa^{2} \nabla \psi^{2}
$$

from which it follows that

$$
P-P_{\mathrm{ref}}=\frac{1}{2} \varepsilon \kappa^{2}\left(\psi^{2}-\psi_{\mathrm{ref}}^{2}\right)
$$



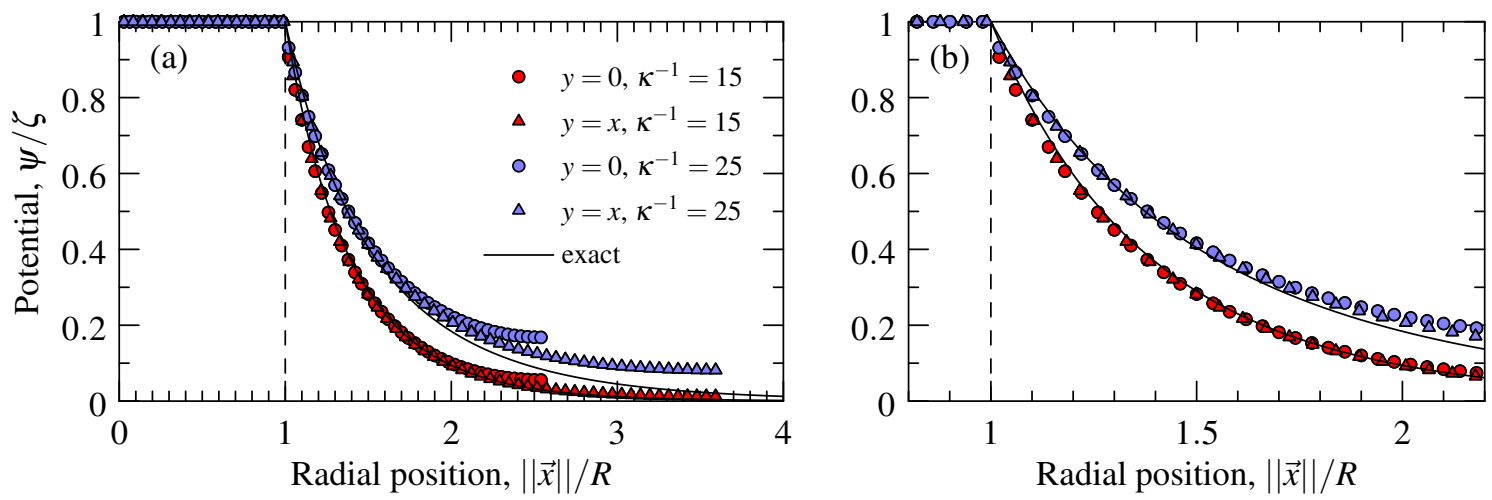

Figure 1: Exact (infinite domain, solid line) and numerical (cubic domain, symbols) solutions for the electrostatic potential around a sphere with a 25 1.u. radius in a $128 \times 128 \times 128$ domain. Results are shown for two Debye lengths $\left(\kappa^{-1}=15:\right.$ red and 25: blue) along two lines in the cross-section through the middle of the sphere: $z=0, y=0$ (circles) and $z=0, y=x$ (triangles). The solutions along the full extents of these lines are shown in (a), while (b) emphasizes the solutions near the surface of the sphere.

where $P_{\text {ref }}$ and $\psi_{\text {ref }}$ are a reference pressure and potential at an arbitrary point in the domain. This is the expected dependence of the osmotic pressure with potential[1]. In Fig. 2 we examine the relationship between the computed static pressure and potential around a sphere in a periodic cubic domain. The reference point was a corner node of the domain, and pressures were computed along the same lines as in Fig. 1 using Eq. 3. For the two simulations shown (with Debye lengths 15 and 25 in a $128 \times 128 \times 128$ domain), bounce-back (no slip) boundaries were implemented for the fluid solver for all internodal links that cross the stair-stepped sphere with radius 25 nodes in the centre of the domain. A single-phase flow was simulated outside the solid surface; the multiphase flow solver was used but with $\phi$ fixed at -1. The agreement between the numerical and exact dependence of the pressure on the potential is good. The slight offset between the two numerical results and the exact trend is attributed to scatter in the values near the chosen reference point. The results for $\varepsilon$ spanning four orders of magnitude were visually indiscernible, and data for only one value $(\varepsilon=0.1)$ are shown.

We now replace the solid spherical boundary with a droplet and consider the equilibrium of a charged drop in electrolyte. Using the exact solution for the potential (Eq. 11), the spherical shell of interfacial charge experiences an outward electrostatic pressure (the product of the surface 


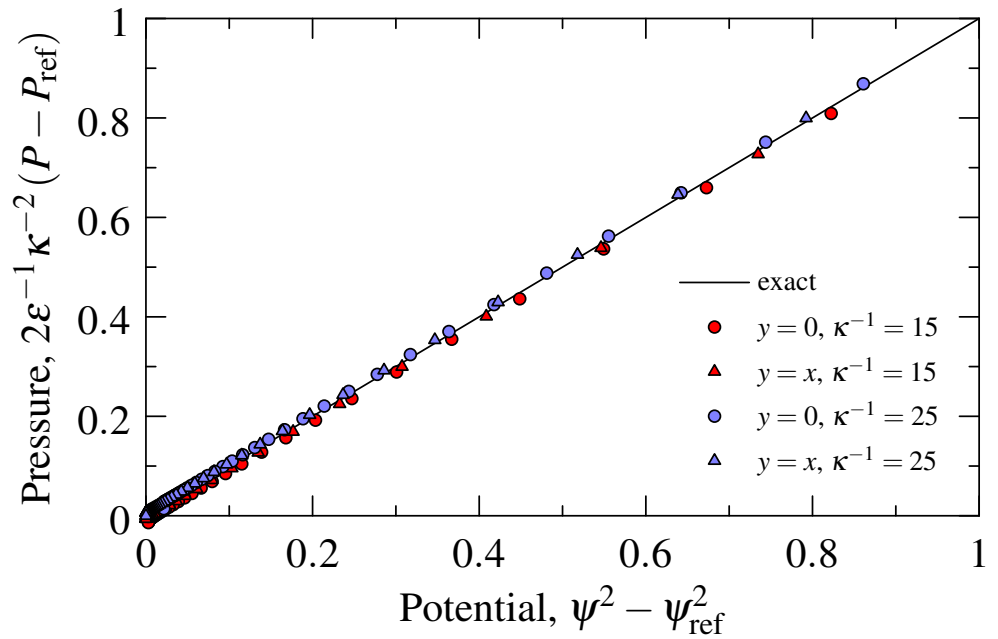

Figure 2: Pressure as a function of electrostatic potential at equilibrium in the electrolyte around a charged sphere (radius 25 l.u.) in a periodic cubic domain $(128 \times 128 \times 128)$. The symbols show the pressures for two Debye lengths $\left(\kappa^{-1}=15:\right.$ red, 25: blue $)$ at the same points from a cross-section through the centre of the droplet as in Fig. 1. The solid line indicates the expected relationship (Eq. 14).

charge, $q_{\mathrm{s}}=-\left.\varepsilon \frac{\mathrm{d} \psi}{\mathrm{d} r}\right|_{r=R}$, and the average of the internal and external electric fields):

$$
\frac{1}{2} q_{\mathrm{s}}\left(-\left.\frac{\mathrm{d} \psi}{\mathrm{d} r}\right|_{r=R^{+}}\right)=\left.\frac{1}{2} \varepsilon \frac{\mathrm{d} \psi}{\mathrm{d} r}\right|_{r=R^{+}} ^{2}=\frac{\varepsilon \zeta^{2}}{2 R^{2}}(\kappa R+1)^{2}
$$

The decrease in pressure inside the drop due to the electrostatic pressure is partially balanced by the rise in pressure outside the drop. From Eq. 14, the pressure at the surface is $\frac{1}{2} \varepsilon \kappa^{2} \zeta^{2}$ higher than infinitely far from the surface. The total difference in pressure between the inside and outside of a drop may be expressed as the sum of the phase field contribution $\Delta P_{\phi}$ and the electrostatic contribution $\Delta P_{\psi}$ such that

$$
\Delta P=\Delta P_{\phi}+\Delta P_{\psi}
$$

where the two components of the pressure change are

$$
\Delta P_{\phi}=\frac{2 \sigma}{R}
$$

and

$$
\Delta P_{\psi}=\frac{1}{2} \varepsilon \kappa^{2} \zeta^{2}-\frac{\varepsilon \zeta^{2}}{2 R^{2}}(\kappa R+1)^{2}=-\frac{\varepsilon \zeta^{2}}{2 R^{2}}(2 \kappa R+1)
$$


We may define an effective interfacial tension $\sigma_{\text {net }}=\frac{1}{2} R \Delta P$ that leads to

$$
\sigma_{\text {net }}=\sigma-\frac{\varepsilon \zeta^{2}}{4 R}(2 \kappa R+1)
$$

In the limit of a small Debye length relative to the drop radius $(\kappa R \gg 1)$, the decrease in the interfacial tension is $\frac{1}{2} \varepsilon \kappa \zeta^{2}$. For physical parameters that create a large decrease (for example, the permittivity of water, a $0.1 \mu \mathrm{m}$ Debye length, and a $100 \mathrm{mV}$ zeta potential), the decrease is $35 \mu \mathrm{N} / \mathrm{m}$, a small fraction of typical $\mathrm{mN} / \mathrm{m}$ interfacial tensions. (We note that with a $100 \mathrm{mV}$ zeta potential, the Debye-Hückel approximation no longer holds at room temperature.)

In the simulations, we consider a wide range of strengths of the electrostatic interactions. We therefore evaluate the accuracy of the pressure difference between the interior of a drop and its surroundings. For this benchmark, we consider a stationary drop with phase field parameters that match the simulations of uncharged collisions with a capillary number of 0.15 and a 25 l.u. drop radius. Keeping the phase field parameters constant, simulations with droplet radii of 25, 37.5, and 50 1.u. were performed in periodic cubic domains with 128, 192, and 256 1.u. sides. For each drop radius, three simulations were performed: one without charge, one with $\kappa^{-1}=15$ l.u., and one with $\kappa^{-1}=25$. In the charged simulations, $\varepsilon \kappa \zeta^{2} / \sigma$ was 0.9 with $\kappa^{-1}=15$ and 0.53 with $\kappa^{-1}=25$. The pressure difference at steady state between the centre of the drop and the corner of the domain is shown in Fig. 3. Good agreement is seen in both the uncharged and charged cases. In the uncharged cases, the pressure differences in the simulations are consistently lower than the theoretical values by at most $3.6 \%$. In the worst charged case, the difference is $4.3 \%$. The parameters of this benchmark were chosen to examine the decrease in pressure difference relative to the uncharged cases, and the strength of electrostatic interactions was significantly higher than it is in most of the simulations we present in the section that follows.

In diffuse interface methods, spurious currents are present at static equilibrium. With the high body forces used in the pressure benchmark, the maximum spurious speed was $5.0 \times 10^{-4}$ (1.u.) while the average in a cross-section through the droplet was $6.6 \times 10^{-6}$ for the worst case. In the simulations without charge, the maximum speed was $2.8 \times 10^{-5}$ and the average was $1.1 \times 10^{-6}$. To assess the magnitude of these spurious currents, we compare them with the characteristic shear speed $\dot{\gamma} R$, where $\dot{\gamma}$ is the shear rate, of the collision simulations we perform at the same conditions. 


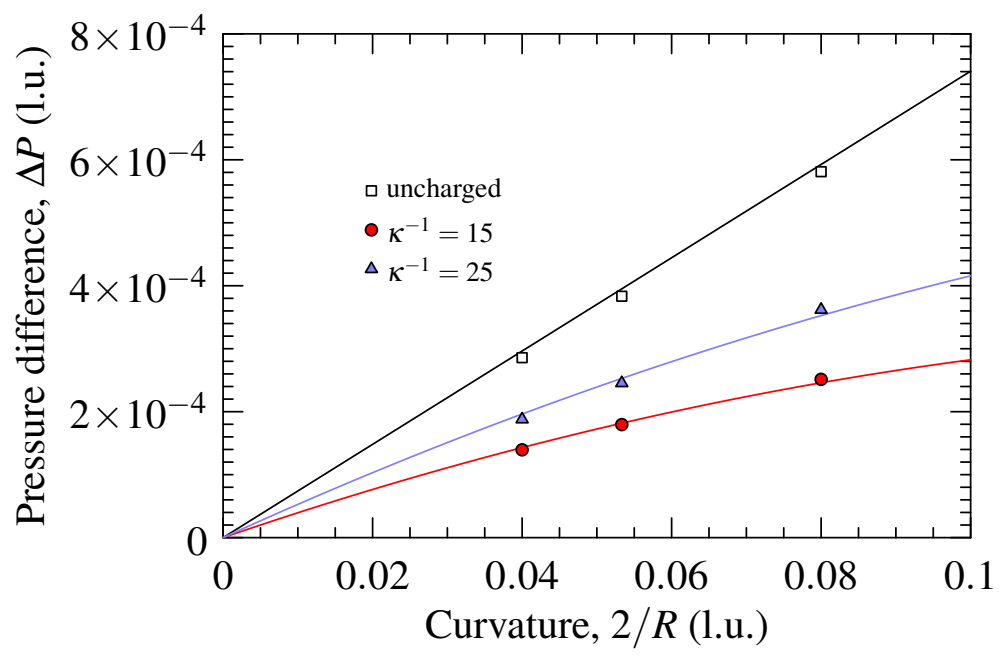

Figure 3: Difference between the internal and external pressures for drops with increasing curvature $2 / R$. The expected relationship (solid lines, Eq. 16) and simulation results (symbols) are shown for uncharged (black squares) and charged drops with two Debye lengths ( $\kappa^{-1}=15$ : red circles, 25: blue triangles).

For the worst charged case, the maximum spurious speed is $7 \%$ of $\dot{\gamma} R$, and the average spurious speed is $0.1 \%$ of $\dot{\gamma} R$. In comparison, the maximum and average spurious speeds are $0.4 \%$ and $0.02 \%$, respectively, in simulations without electric body forces. Though spurious currents are an order of magnitude higher in the simulations with charge, they are low compared to the flow speeds we impose to shear the droplets.

We briefly point out that the pressure difference given by Eq. 16 is negative when the electrostatic contribution exceeds the contribution from the phase field. Such cases are easy to implement in the simulations by specifying a sufficiently low interfacial tension $\sigma$. Under these conditions, the interface is unstable, and the drop breaks apart. This outcome is similar to the Rayleigh instability of charged drops in air[35, 36], but we do not study this phenomenon further in this paper.

Overall, the benchmark simulations indicate high accuracy for the potential solution and coupling with the flow. We consider next the simulations of charged droplet collisions.

\subsection{Droplet collisions}

Simulations of collisions between charged droplets in shear were performed to determine the effects of the strength of electrostatic repulsion on the critical conditions for coalescence. These simulations were performed under conditions identical to those in our previous study of uncharged 
droplets[30]. Two shear planes moving in opposite directions are separated by a height $H$ (in $y$, the velocity gradient direction) in a domain with a width $W=H$ (in $z$, the vorticity direction) and a length $L=2 H$ (in $x$, the shear direction). Two droplets with radius $R$ are placed in the middle of the domain, with their centres separated by a vertical offset $\Delta Y /(2 R)=0.86$ and a horizontal distance $\Delta X /(2 R)=1.26$. The confinement of the drops is $2 R / H=0.39$. The two fluids have equal densities and viscosities. The domain is periodic in the $x$ direction, and reflection boundary conditions are specified at the ends of the domain in the $z$ direction. On the shear planes, the flow velocity is the shear velocity, and a reflection condition is used for the phase field. For the simulations with charge, the potential is periodic in the $x$ direction and has reflection (zero charge) conditions on the other planes. As before[30], we use internal symmetry and rotational symmetry boundary conditions through the centres of the drops to reduce the computed domain size to one quarter of the full $L \times W \times H$ system.

In all simulations of droplet collisions in shear, the shear rate $\dot{\gamma}$ was specified such that the Reynolds number was $\operatorname{Re}=\frac{\dot{\gamma} R^{2}}{v}=1$. As in previous work[30, 31], we use $\operatorname{Re}=1$ as a computationally efficient surrogate for much lower Re. The phase field parameters were chosen to have well-resolved interfaces with $\ell_{\phi}=2$. When interfaces are resolved, coalescence is a consequence of the dynamics of the phase field rather than inadequate resolution of interfaces and films between drops[30]. The Péclet number that characterizes the phase field mobility, $\mathrm{Pe}=\frac{\dot{\gamma} R \ell_{\phi}}{M A}$ was fixed at $10[30,31]$. The interfacial tension between the drop and bulk phases was varied to study the effect of the capillary number $\mathrm{Ca}=\frac{\rho v \dot{\gamma} R}{\sigma}$.

Droplets coalesce unless the capillary number exceeds a critical value that is affected by the ratio of the drop radius and interface thickness[30]. For fixed capillary and Reynolds numbers, a larger radius relative to the interface thickness suppresses coalescence. Therefore, critical capillary numbers decrease with increasing $R / \ell_{\phi}$. The rate of this decrease was previously studied[30] by performing simulations with increasing droplet (and domain) sizes while keeping the interface thickness constant. We now study the effects of electrostatic repulsion on the relationship between the critical capillary number and droplet size.

The inclusion of electrostatic interactions between charged droplets through an electrolyte in- 
troduces two non-dimensional parameters. The first parameter is the ratio of the Debye length and a characteristic length for which two choices are meaningful: the drop radius and interface thickness. The resulting ratios are $\kappa R$ and $\kappa \ell_{\phi}$, respectively. For the second parameter, which characterizes the strength of electric forces, we choose the ratio of electric and viscous forces $\frac{\varepsilon \zeta^{2}}{\rho v^{2}}$. In the simulation parameter space, the zeta potential, density, and viscosity are all fixed, leading to $\frac{\varepsilon \zeta^{2}}{\rho v^{2}}=36 \varepsilon$. While in physical experiments the strength of electric interactions usually varies due to $\zeta$, in the simulations potentials are normalized with respect to $\zeta$ and we vary $\varepsilon$.

Figure 4 illustrates sample collisions between uncharged and charged droplets. In these simulations, the droplet radius was 50 l.u., the largest we consider in this work, and the computed domain was $512 \times 128 \times 128$. Running on one NVIDIA Tesla M2070 GPU, the uncharged simulation completed 240000 timesteps in 16 hours at a speed of 34.9 million lattice updates per second (Mlups). The speed of simulations with charge depends on the number of iterations required for the potential to converge. For the charged simulation shown in Fig. 4, the effective speed was 2.8 Mlups with an average of 147 iterations of the potential solver at each timestep. Though 12.5 times slower than the uncharged simulation, this simulation running on a GPU is faster than an implementation of the free-energy LBM (without charge) on 8 conventional CPUs that ran at 2 Mlups[54]. Comparing the first two simulations presented in Fig. 4, we see that the repulsion between the charged droplets prevents coalescence at $\mathrm{Ca}=0.06$. When $\mathrm{Ca}$ is lowered to 0.055 while keeping the other parameters of the charged system constant, which is shown as the third simulation in Fig. 4, the droplets coalesce. The shapes of the droplets before and after coalescence evolve as in the uncharged case, except that coalescence occurs slightly earlier.

The effects of the Debye length and the strength of the electrostatic interactions on the outcomes of droplet collisions are shown in Fig. 5. To imitate physical experiments with the same fluid system and increasing droplet radii, the Debye length was kept constant while the droplet radius and domain size were increased in the same proportion. Simulations were performed with $\frac{\varepsilon \zeta^{2}}{\rho v^{2}}$ from $3.6 \times 10^{-4}$ to 3.6 (based on choosing $\varepsilon$ from $10^{-5}$ to $10^{-1}$ in lattice units). The simulations of uncharged collisions were repeated (instead of using previous results[30]) to include an equilibration delay because the simulations with charge included a short delay for interface equi- 


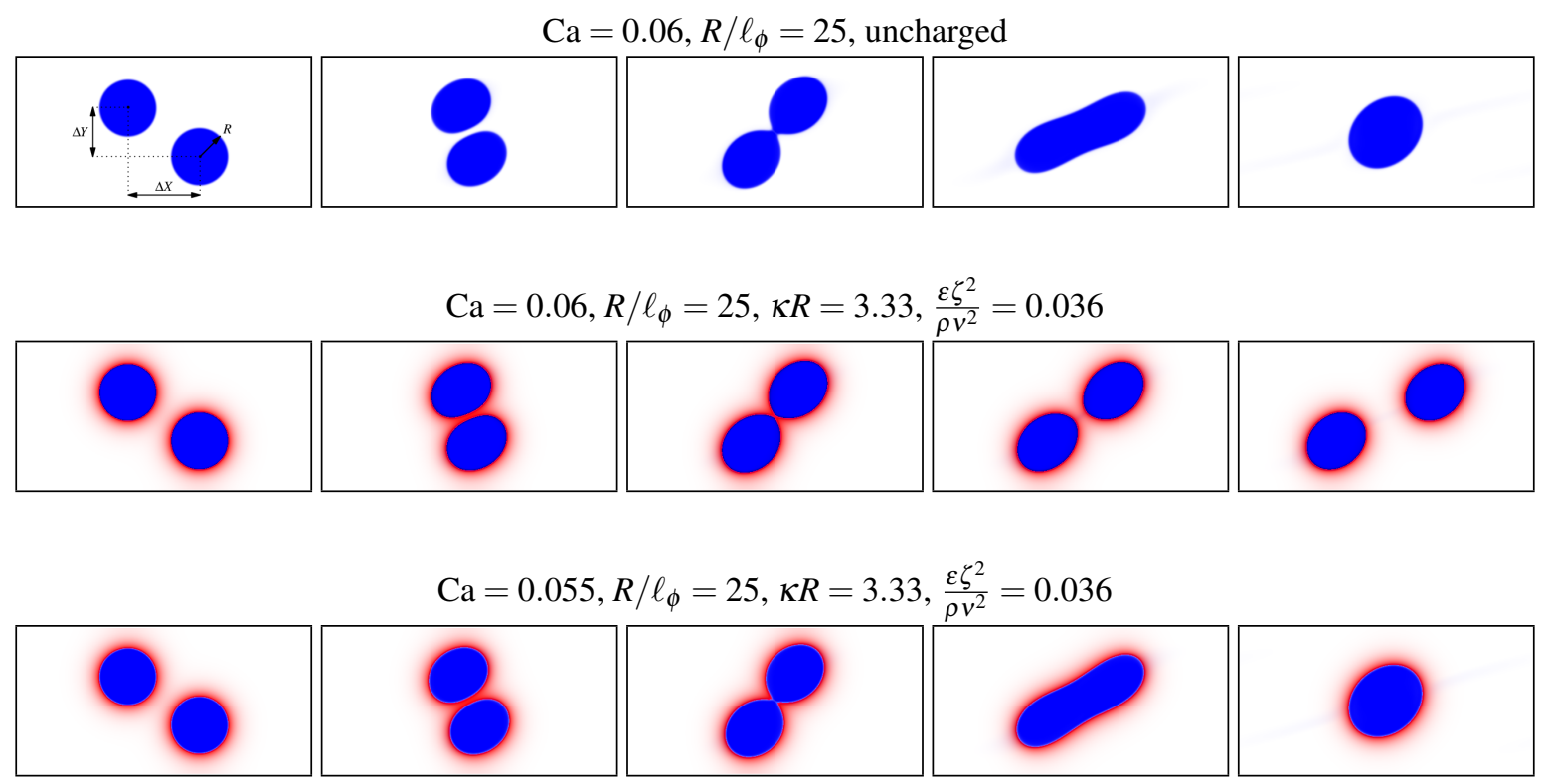

Figure 4: Sample collisions of uncharged (upper sequence) and charged (lower two sequences) droplets. The colour ranges from white $(\phi=-1)$ to blue $(\phi=1)$ for the phase field and white $(\psi=0)$ to red $(\psi=\zeta)$ for the potential outside the drops. The non-dimensional times of each frame are $\dot{\gamma} t=0,2,3.2,4.7,6.7$ (upper sequence), $\dot{\gamma} t=0,2$, 3.2, 3.6, 4.3 (middle sequence), and $\dot{\gamma} t=0,1.9,3.1,4,6$ (bottom sequence).

libration before shearing is started. As would be expected, this delay did not change the critical capillary numbers. At the conditions of the simulations, two critical capillary numbers separate the capillary number ranges for three possible outcomes. Below the lower critical capillary number, the droplets coalesce, while above the upper critical capillary number the droplets slide over each other. Between the two critical $\mathrm{Ca}$, the interfaces of the droplets temporarily merge, but the bridge breaks because the drops are sheared apart faster than the bridge grows[30]. The details of the breakup of the bridge in higher-resolution simulations are reported in [31]. Capillary numbers were systematically searched until the interval containing each critical capillary number was narrowed to 0.005 . In the cases with the strongest electric interactions, coalescence was not observed, and a symbol is shown for the lowest capillary number that could be simulated before numerical instability occurred. This instability is attributed to the high interfacial tensions needed to achieve low capillary numbers.

Figure 5 shows that the critical capillary numbers for coalescence shift downward with increasing $\varepsilon \zeta^{2} / \rho v^{2}$ and the decrease is smaller for the longer Debye length. This means that a slower 

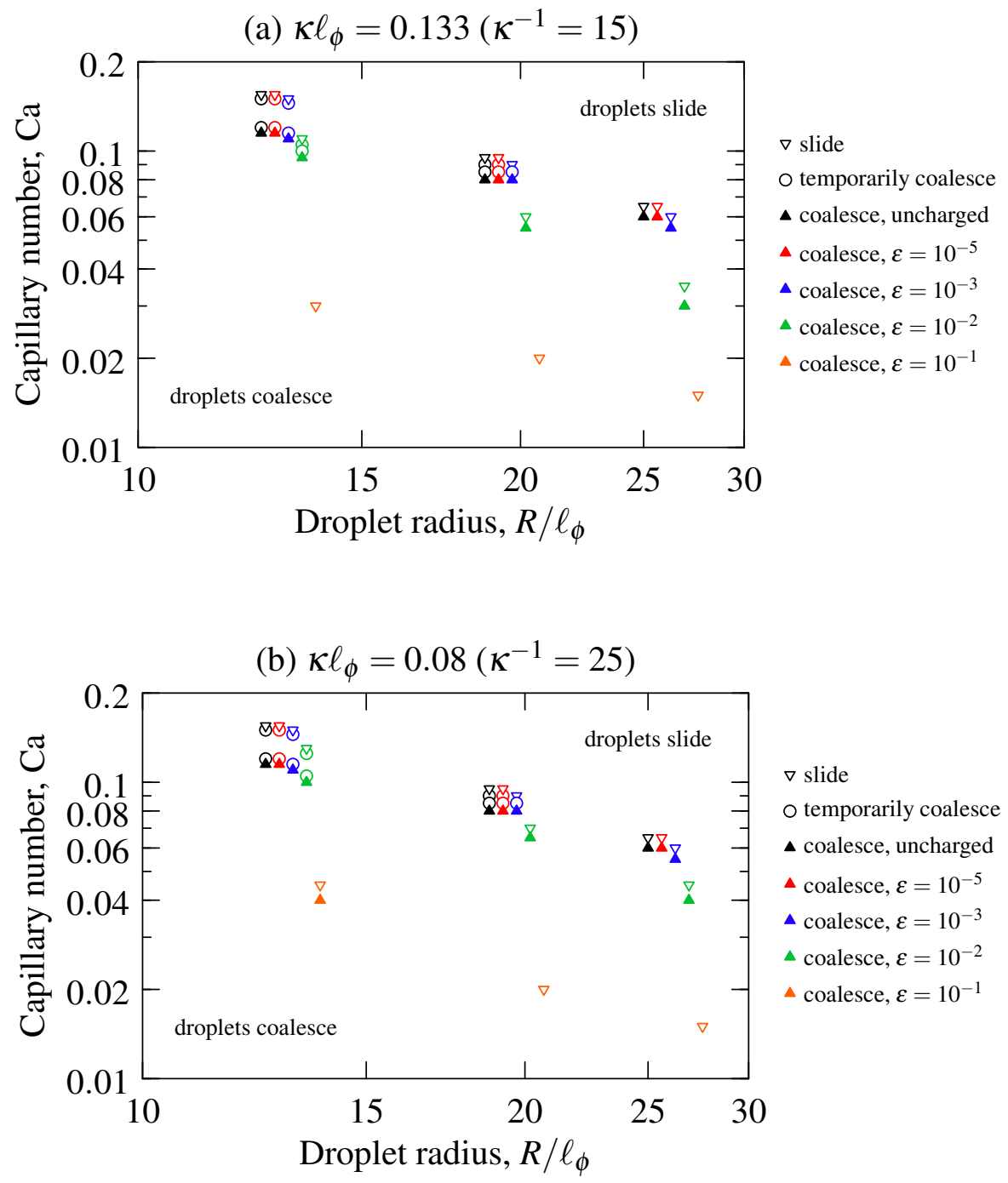

Figure 5: Outcomes of binary droplet collisions at varying capillary numbers $\mathrm{Ca}$, droplet radii $R$ relative to the characteristic interface thickness $\ell_{\phi}$, and several strengths of the electrostatic interactions (uncharged and $\kappa^{-1}=$ (a) 15 and (b) 25 1.u. with $\varepsilon=10^{-1}, 10^{-2}, 10^{-3}, 10^{-5}$ 1.u.). In each cluster of data points with different $\varepsilon$, the radius is the same as in the uncharged case, but the symbols have been offset horizontally to separate them. Only the simulation results that are closest to a critical capillary number are shown: open triangles indicate the lowest capillary number at which sliding was observed, open circles indicate capillary numbers at which coalescence is temporary, and filled triangles indicate the highest capillary numbers at which the droplets coalesce. 
shear rate is needed to coalesce charged drops than uncharged drops. Electrostatic repulsion, therefore, has the expected effect of suppressing coalescence because droplets now slide at lower shear rates. To interpret these outcomes, we consider the force per unit area $f_{\mathrm{p}}$ on two parallel plates with equal potential separated by a distance $d[1]$ :

$$
f_{\mathrm{p}}=\frac{\varepsilon \kappa^{2} \zeta^{2}}{2 \cosh ^{2}(\kappa d / 2)}
$$

For a compressed pair of colliding droplets, we choose a separation distance equal to the thickness of the phase field interface, $d=5 \ell_{\phi}=10$ 1.u.. At this separation, $f_{p}$ is maximum when $\kappa^{-1} \approx$ $0.42 d=4.21$.u. and decreases with increasing Debye length (decreasing $\kappa$ ). This explains the weaker repulsion of droplets with thicker EDLs. Due to the flattening of drops during collisions (Fig. 4), we estimate the force on the drops as $\pi R^{2} f_{\mathrm{p}}$ rather than being proportional to $\pi R \varepsilon \kappa \zeta^{2}$ for spheres in the Derjaguin limit[1]. To determine when electrostatic forces affect droplet collisions, we therefore compare $f_{p}$ with the viscous stress $\rho v \dot{\gamma}$ and form the ratio

$$
\frac{\varepsilon \kappa^{2} \zeta^{2}}{\rho v \dot{\gamma}}
$$

We have omitted the proportionality constant $\frac{1}{2}$ and also the factor $\cosh ^{2}(\kappa d / 2)$ because $\cosh (\kappa d / 2) \approx$ 1 for the parameters we consider. As shown in Fig. 6, the critical capillary number decreases as this ratio increases. Electrostatic forces change the critical capillary numbers for coalescence once $\frac{\varepsilon \kappa^{2} \zeta^{2}}{\rho v \dot{\gamma}} \approx 1$. With increasing $R$ and fixed physical properties, the maximum shear rate for coalescence decreases and the relative magnitude of electric forces at near critical conditions increases. Presumably, sufficiently large droplets cannot coalesce because electric repulsion is too high compared to shear stress at the low shear rates required for coalescence. In the simulations, we resolve the Debye length (and interface thickness), and the droplets are not large enough to study the behaviour at this limit. In fact, considering the high critical capillary numbers at a high initial vertical offset between the drops (in comparison with experiments[28]), the droplets we simulate are quite small.

To compare the simulations with an aqueous system, we first estimate the physical size of the droplets through the ratio of the droplet radius and minimum stable film thickness before coalescence, estimated to be $3 \ell_{\phi}=61$.u. for simulations with uncharged interfaces[30]. Taking 


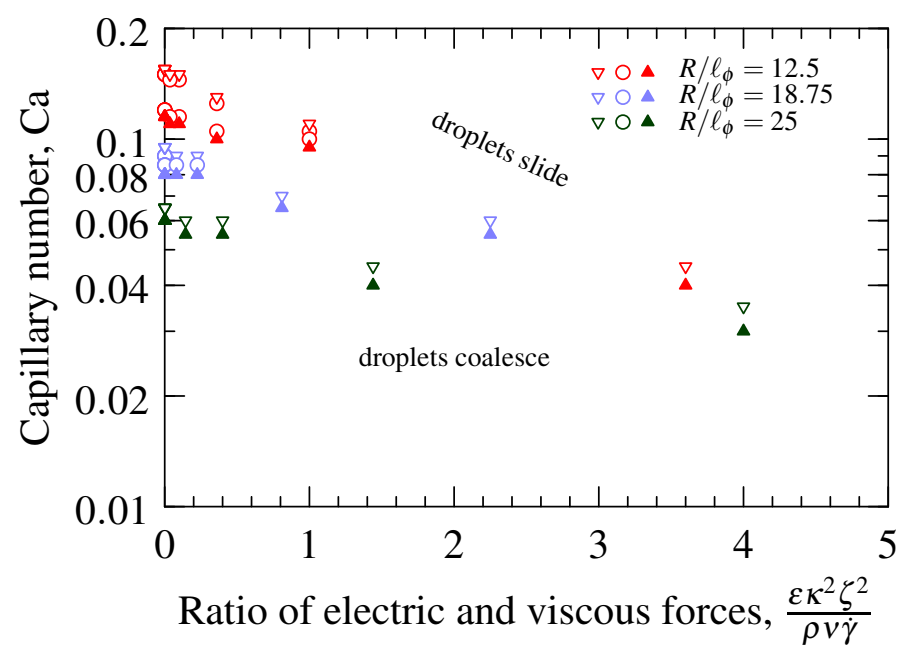

Figure 6: Effect of the ratio of electric and viscous forces on the critical capillary numbers for coalescence. The symbols have the same meaning as in Fig. 5 but are coloured by the droplet radius: $R / \ell_{\phi}=12.5$ in red, $R / \ell_{\phi}=18.75$ in blue, and $R / \ell_{\phi}=25$ in green.

a physical critical film thickness on the order of $10 \mathrm{~nm}$, the physical radius of the $R=251 . \mathrm{u}$. droplets is estimated to be $42 \mathrm{~nm}$, and $83 \mathrm{~nm}$ for the drops with $R=501$.u., both in the range of microemulsions[64]. The Debye lengths are then $25 \mathrm{~nm}$ and $42 \mathrm{~nm}$ for $\kappa^{-1}=15$ and 25 l.u., respectively, which are reasonable for dilute aqueous systems[1]. Using the larger drop radius and shorter Debye length with the permittivity, density, and viscosity of water, the condition for a noticeable change in critical capillary numbers becomes

$$
\frac{\varepsilon \kappa^{2} \zeta^{2}}{\rho v \dot{\gamma}} \approx 1 \Rightarrow \zeta \approx 360 \mathrm{mV}
$$

which is higher than typical zeta potentials, e.g. up to $|\zeta|=120 \mathrm{mV}$ for hexane in $1 \mathrm{mM} \mathrm{KCl}$ solution[6]. At these high zeta potentials the Debye-Hückel approximation becomes poor, and the effects of finite ion size need to be considered[1]. In comparison, the required potential is $25 \mathrm{mV}$ for a droplet with $R=1.2 \mu \mathrm{m}$ at the same conditions.

For the points shown in Figs. 5 and 6, the difference between $\sigma$ and $\sigma_{\text {net }}$ is at most $20 \%$ for the points with $\varepsilon=0.1$, less than $5 \%$ for the points with $\varepsilon=0.01$, and less than $1 \%$ for all other points. The change in the conditions for coalescence cannot therefore be attributed to changes in the effective interfacial tension of the drops and therefore their capillary numbers (which was 
defined using $\sigma$ rather than $\sigma_{\text {net }}$ ).

The colliding droplets in the simulations have constant surface potentials. Since surface charges are proportional to the local normal potential gradient, surface charges decrease as the proximity to another (equal potential) interface decreases. Such a decrease in charge on approaching interfaces would not occur with constant-charge interfaces, causing a stronger repulsion between the droplets. In the limit of small separation distance relative to the Debye length, the force on parallel plates with constant surface potential, Eq. 20, simplifies to $\frac{1}{2} \varepsilon \kappa^{2} \zeta^{2}+O\left(d^{2}\right)$. In contrast, the force on plates with constant charge[1] diverges as $d^{-2}$. Due to this divergence, constant surface charges are not expected to be maintained at small separations in real systems, and constant potential behaviour is likely for small $d[1]$. Simulations with constant surface charge and other charge regulation models $[1,57]$ are areas for future investigation.

\section{Concluding remarks}

Simulations of charged droplet collisions in shear were performed by coupling a phase field method for two-component flows with an iterative finite difference solver for the electrostatic potential in the EDL around the deforming drops. A benchmark study of transient electroosmotic flow in a square channel was used to assess the accuracy of the coupled solvers for single phase flow. A static benchmark of the pressure differences across charged and uncharged droplet interfaces was used to evaluate the accuracy of the forcing in the multiphase solver.

Collisions of droplets with constant surface potential and electric forces spanning four orders of magnitude were performed to quantify the change in critical capillary number for coalescence as a function of the strength of the electrostatic repulsion between the droplets. Electric forces begin to affect the critical capillary numbers once they are comparable to viscous forces. Increasing electric forces decrease the critical capillary number for coalescence, and the decrease is greater for shorter Debye lengths. Since the simulations resolve the Debye length and the film between the droplets, the droplet radii are only several times larger than these length scales. Consequently, the effective physical radii of the droplets we simulate are between 10 and $100 \mathrm{~nm}$, as found in microemulsions. For droplets of this size at the simulated conditions, electric repulsion is weak compared to shear forces. In systems with larger droplets and lower shear rates, electric repulsion 
would be more significant. Furthermore, at the low Reynolds numbers we presently consider, ion advection is negligible. For higher Reynolds numbers (based on the imposed shear rate) or fast post-coalescence flows, such as when the droplets' sizes and therefore their internal pressures differ, ion convection, diffusion, and migration must all be considered together.

Several areas for future work are available towards the goals of developing accurate simulations of the electric phenomena that contribute to interfacial interactions in multiphase flows and understanding their impact on larger scales of such flows. The simulations may be developed further by solving the non-linear Poisson-Boltzmann equation, including external electric fields and modelling leaky dielectrics[44], solving Nernst-Planck advection-diffusion equations for ion concentrations, and implementing constant surface charge and charge regulation boundary conditions on fluid interfaces. To simulate flows with larger droplets, the simulation methods can be adapted to use multiple GPUs in parallel, as has been done for simulations without charge[30, 31]. Such simulations could be used to study droplet interactions in a flowing emulsion. The simulation methods may also be adapted to study other electric phenomena in fluids, such as the Rayleigh instability, droplet electrophoresis, and electrowetting.

\section{Acknowledgments}

This research has been enabled by the use of computing resources provided by WestGrid, the Shared Hierarchical Academic Research Computing Network (SHARCNET: www . sharcnet . ca), and Compute/Calcul Canada. O.S. thanks NSERC for an Alexander Graham Bell Canada Graduate Scholarship. 


\section{References}

[1] J. H. Masliyah, S. Bhattacharjee, Electrokinetic and Colloid Transport Phenomena, WileyInterscience, 2006.

[2] H. McTaggart, The electrification at liquid-gas surfaces, Philos. Mag. 27 (1914) 297-314.

[3] J. Beattie, A. Djerdjev, G. Warr, The surface of neat water is basic, Faraday Discuss. 141 (2009) 31-39.

[4] J. Carruthers, The electrophoresis of certain hydrocarbons and their simple derivatives as a function of pH, T. Faraday Soc. 34 (1938) 300-307.

[5] W. Dickinson, The effect of $\mathrm{pH}$ upon the electrophoretic mobility of emulsions of certain hydrocarbons and aliphatic halides, T. Faraday Soc. 37 (1941) 140-148.

[6] J. Liu, Z. Zhou, Z. Xu, Electrokinetic study of hexane droplets in surfactant solutions and process water of bitumen extraction systems, Ind. Eng. Chem. Res. 41 (2002) 51-57.

[7] M. Mooney, Variations in the cataphoretic mobilities of oil drops in water, Phys. Rev. 23 (1924) 396-411.

[8] F. Booth, The cataphoresis of spherical fluid droplets in electrolytes, J. Chem. Phys. 19 (1951) $1331-1336$.

[9] A. Taylor, F. Wood, The electrophoresis of hydrocarbon droplets in dilute solutions of electrolytes, T. Faraday Soc. 53 (1957) 523-529.

[10] S. Levine, R. O'Brien, A theory of electrophoresis of charged mercury drops in aqueous electrolyte solution, J. Colloid Interf. Sci. 43 (1973) 616-629.

[11] J. Baygents, D. Saville, Electrophoresis of drops and bubbles, J. Chem. Soc. Faraday Trans. 87 (1991) 1883-1898. 
[12] B. Derjaguin, L. Landau, Theory of the stability of strongly charged lyophobic sols and of the adhesion of strongly charged particles in solution of electrolytes, Acta Physicochim. (URSS) 14 (1941) 633-662.

[13] E. Verwey, J. Overbeek, Theory of the Stability of Lyophobic Colloids, Elsevier, 1948.

[14] J. Drelich, J. Long, A. Yeung, Determining surface potential of the bitumen-water interface at nanoscale resolution using atomic force microscopy, Can. J. Chem. Eng. 85 (2008) 625-634.

[15] P. Esmaeili, F. Lin, A. Yeung, Stability of emulsified heavy oil: The combined effects of deterministic DLVO forces and random surface charges, Langmuir 28 (2012) 4948-4954.

[16] D. Saville, The effects of interfacial tension gradients on the motion of drops and bubbles, Chem. Eng. J. 5 (1973) 251-259.

[17] H. Liu, Y. Zhang, Phase-field modeling droplet dynamics with soluble surfactants, J. Comput. Phys. 229 (2010) 9166-9187.

[18] G. Tryggvason, B. Bunner, A. Esmaeeli, D. Juric, N. Al-Rawahi, W. Tauber, J. Han, S. Nas, Y.-J. Jan, A front-tracking method for the computations of multiphase flow, J. Comp. Phys. 169 (2001) 708-759.

[19] S. Quan, J. Lou, D. Schmidt, Modeling merging and breakup in the moving mesh interface tracking method for multiphase flow simulations, J. Comp. Phys. 228 (2009) 2660-2675.

[20] G. Tryggvason, R. Scardovelli, S. Zaleski, Direct Numerical Simulations of Gas-Liquid Multiphase Flows, Cambridge University Press, 2011.

[21] C. Tsouris, L. Tavlarides, Breakage and Coalescence Models for Drops in Turbulent Dispersions, AIChE Journal 40 (1994) 395-406.

[22] P. Perlekar, L. Biferale, M. Sbragaglia, S. Srivastava, F. Toschi, Droplet size distribution in homogeneous isotropic turbulence, Phys. Fluids 24 (2012) 065101. 
[23] V. Cristini, Y.-C. Tan, Theory and numerical simulation of droplet dynamics in complex flows — a review, Lab Chip 4 (2004) 257-264.

[24] Y. Hu, D. Pine, L. Leal, Drop deformation, breakup, and coalescence with compatibilizer, Phys. Fluids 12 (3) (2000) 484-489.

[25] H. Yang, C. Park, Y. Hu, L. Leal, The coalescence of two equal-sized drops in a twodimensional linear flow, Phys. Fluids 13 (2001) 1087-1106.

[26] L. Leal, Flow induced coalescence of drops in a viscous fluid, Phys. Fluids 16 (2004) 18331851.

[27] Y. Yoon, M. Borrell, C. Park, L. Leal, Viscosity ratio effects on the coalescence of two equalsized drops in a two-dimensional linear flow, J. Fluid Mech. 525 (2005) 355-379.

[28] D. Chen, R. Cardinaels, P. Moldenaers, Effect of confinement on droplet coalescence in shear flow, Langmuir 25 (22) (2009) 12885-12893.

[29] P. De Bruyn, R. Cardinaels, P. Moldenaers, The effect of geometrical confinement on coalescence efficiency of droplet pairs in shear flow, J. Colloid Interf. Sci. 409 (2013) 183-192.

[30] O. Shardt, J. Derksen, S. Mitra, Simulations of droplet coalescence in simple shear flow, Langmuir 29 (2013) 6201-6212.

[31] O. Shardt, S. Mitra, J. Derksen, The critical conditions for coalescence in phase field simulations of colliding droplets in shear, Langmuir 30 (2014) 14416-14426.

[32] A. Briant, J. Yeomans, Lattice Boltzmann simulations of contact line motion. II. Binary fluids, Phys. Rev. E 69 (2004) 031603.

[33] P. Yue, J. Feng, C. Liu, J. Shen, Diffuse-interface simulations of drop coalescence and retraction in viscoelastic fluids, J. Non-Newtonian Fluid Mech. 129 (2005) 163-176. 
[34] B. Dollet, A. Scagliarini, M. Sbragaglia, Two-dimensional plastic flow of foams and emulsions in a channel: experiments and lattice Boltzmann simulations, J. Fluid Mech. 766 (2015) $556-589$.

[35] T. Matsuyama, T. Abe, H. Yamamoto, Lattice Boltzmann method study of Rayleigh instability of a charged droplet, Advanced Powder Technol. 18 (2007) 93-104.

[36] L. Rayleigh, On the equilibrium of liquid conducting masses charged with electricity, Philos. Mag. 14 (1882) 184-186.

[37] J. Wang, M. Wang, Z. Li, Lattice Poisson-Boltzmann simulations of electro-osmotic flows in microchannels, J. Colloid Interf. Sci. 296 (2006) 729-736.

[38] M. Wang, Q. Kang, Modeling electrokinetic flows in microchannels using coupled lattice Boltzmann methods, J. Comput. Phys. 229 (2010) 728-744.

[39] T.-Y. Lin, C.-L. Chen, Analysis of electroosmotic flow with periodic electric and pressure fields via the lattice-Poisson-Boltzmann method, Appl. Math. Model. 37 (2013) 2816-2829.

[40] D. Hlushkou, D. Kandhai, U. Tallarek, Coupled lattice-Boltzmann and finite-difference simulation of electroosmosis in microfluidic channels, Int. J. Numer. Meth. Fluids 46 (2004) $507-532$.

[41] M. Wang, N. Pan, J. Wang, S. Chen, Lattice Poisson-Boltzmann Simulations of Electroosmotic Flows in Charged Anisotropic Porous Media, Communications in Computational Physics 2 (6) (2007) 1055-1070.

[42] J. López-Herrera, S. Popinet, M. Herrada, A charge-conservative approach for simulating electrohydrodynamic two-phase flows using volume-of-fluid, J. Comput. Phys. 230 (5) (2011) 1939-1955.

[43] K. Teigen, S. Munkejord, Influence of surfactant on drop deformation in an electric field, Phys. Fluids 22 (2010) 112104. 
[44] Y. Lin, P. Skjetne, A. Carlson, A phase field model for multiphase electro-hydrodynamic flow, Int. J. Multiphase Flow 45 (2012) 1-11.

[45] B. Ben-Yaakov, D. Andelman, R. Podgornik, D. Harries, Ion-specific hydration effects: Extending the Poisson-Boltzmann theory, Curr. Opin. Colloid Interface Sci. 16 (2011) 542-550.

[46] A. Onuki, Ginzburg-Landau theory of solvation in polar fluids: Ion distribution around an interface, Phys. Rev. E 73 (2006) 021506.

[47] R. G. M. van der Sman, M. B. J. Meinders, Mesoscale models of dispersions stabilized by surfactants and colloids, Adv. Colloid Interface Sci. 211 (2014) 63-76.

[48] B. Rotenberg, I. Pagonabarraga, D. Frenkel, Coarse-grained simulations of charge, current, and flow in heterogeneous media, Faraday Discuss. 144 (2009) 223-243.

[49] X. He, Q. Zou, L.-S. Luo, M. Dembo, Analytic solutions of simple flows and analysis of nonslip boundary conditions for the lattice Boltzmann BGK model, J. Stat. Phys. 87 (1997) $115-136$.

[50] C. Pooley, K. Furtado, Eliminating spurious velocities in the free-energy lattice Boltzmann method, Phys. Rev. E 77 (2008) 046702.

[51] S. Chen, G. Doolen, Lattice Boltzmann method for fluid flows, Annu. Rev. Fluid Mech. 30 (1998) 329-364.

[52] C. Aidun, J. Clausen, Lattice-Boltzmann Method for Complex Flows, Annu. Rev. Fluid Mech. 42 (2010) 439-472.

[53] S. van der Graaf, T. Nisisako, C. Schroën, R. van der Sman, R. Boom, Lattice Boltzmann Simulations of Droplet Formation in a T-Shaped Microchannel, Langmuir 22 (2006) 41444152.

[54] A. Komrakova, O. Shardt, D. Eskin, J. Derksen, Lattice Boltzmann simulations of drop deformation and breakup in shear flow, Int. J. Multiphase Flow 59 (2014) 24-43. 
[55] S. Wall, The history of electrokinetic phenomena, Curr. Opin. Colloid In. 15 (2010) 119-124.

[56] S. Carnie, D. Chan, Interaction free energy between identical spherical colloidal particles: The linearized Poisson-Boltzmann theory, J. Colloid Interf. Sci 155 (1993) 297-312.

[57] S. Carnie, D. Chan, J. Stankovich, Computation of forces between spherical colloidal particles: Nonlinear Poisson-Boltzmann theory, J. Colloid Interf. Sci 165 (1994) 116-128.

[58] P. Das, S. Bhattacharjee, W. Moussa, Electrostatic double layer force between two spherical particles in a straight cylindrical capillary: Finite element analysis, Langmuir 19 (2003) 4162-4172.

[59] T. Squires, M. Bazant, Induced-charge electro-osmosis, J. Fluid Mech. 509 (2004) 217-252.

[60] M. Dowle, R. Mantel, D. Barkley, Fast simulations of waves in three-dimensional excitable media, Int. J. Bifurcat. Chaos 7 (1997) 2529-2545.

[61] P. Moin, Fundamentals of Engineering Numerical Analysis, Cambridge University Press, 2nd edn., 2010.

[62] E. Purcell, D. Morin, Electricity and Magnetism, Cambridge University Press, 3rd edn., 2013.

[63] D. Jacqmin, Calculation of two-phase Navier-Stokes flows using phase-field modeling, J. Comput. Phys. 155 (1) (1999) 96-127.

[64] L. L. Schramm, Emulsions, Foams, and Suspensions, Wiley-VCH, 2005. 